\title{
Intraoperative use of diffusion tensor imaging fiber tractography and subcortical mapping for resection of gliomas: technical considerations
}

\author{
Lorenzo Bello, M.D., ${ }^{1,2}$ Antonella Castellano, M.D., ${ }^{3,4}$ Enrica Fava, M.D., 1 \\ Giuseppe Casaceli, M.D., ${ }^{1}$ Marco Riva, M.D., ${ }^{1}$ Giuseppe ScotTi, M.D., ${ }^{3}$ \\ Sergio Maria Gaini, M.D., ${ }^{1}$ and Andrea Falini, M.D. ${ }^{3}$
}

${ }^{1}$ Division of Neurosurgery, Department of Neurological Sciences, and ${ }^{4}$ Institute of Radiological Sciences, Università degli Studi di Milano; ${ }^{2}$ Fondazione IRCCS Ca' Granda, Ospedale Maggiore Policlinico; and ${ }^{3}$ Neuroradiology, Head and Neck Department and CERMAC (Centro di Eccellenza Risonanza Magnetica ad Alto Campo), San Raffaele Scientific Institute and Vita-Salute University, Milan, Italy

\begin{abstract}
Resection of lesions involving motor or language areas or pathways requires the intraoperative identification of functional cortical and subcortical sites for effectively and safe guidance. Diffusion tensor (DT) imaging and fiber tractography are MR imaging techniques based on the concept of anisotropic water diffusion in myelinated fibers, which enable 3D reconstruction and visualization of white matter tracts and provide information about the relationship of these tracts to the tumor mass. The authors routinely used DT imaging fiber tractography to reconstruct various tracts involved in the motor and/or language system in a large series of patients with lesions involving the motor and/or language areas or pathways. The DT imaging fiber tractography data were loaded into the neuronavigational system and combined intraoperatively with those obtained from direct electrical stimulation applied at the subcortical level. In this paper the authors report the results of their experience, describing the findings for each tract and discussing technical aspects of the combined use as well as the pitfalls. (DOI: 10.3171/2009.12.FOCUSO9240)
\end{abstract}

KEY WoRdS - intraoperative mapping diffusion tensor imaging fiber tractography

- glioma -

- motor and language subcortical sites

$\mathrm{S}$ URGICAL removal of lesions located in or within functional areas requires the intraoperative identification of functional sites at both cortical and subcortical levels to safely resect the tumor and at the same time preserve functional integrity for the patient. ${ }^{10,16,49}$ Commonly referred to as brain mapping, this identification process involves a number of surgical, neurophysiological, and neuropsychological adjuncts, which work together to provide the functional results.,.$^{3,9,15}$ Neuroradiological techniques, such as fMR imaging and DT imaging-FT, respectively, offer the opportunity to identify preoperatively the location of functional sites at a corti-

\footnotetext{
Abbreviations used in this paper: CST = corticospinal tract; DES $=$ direct electrical stimulation; DICOM = Digital Imaging and Communications in Medicine; DT = diffusion tensor; ECoG = electrocorticography; $\mathrm{EEG}=$ electroencephalography; $\mathrm{EMG}=$ electromyography; fMR = functional MR; FOV = field of view; $\mathrm{FT}=$ fiber tractography; IFO = inferior frontooccipital fasciculus; $\mathrm{ILF}=$ inferior longitudinal fasciculus; $\mathrm{MEP}=$ motor evoked potential; ROI = region of interest; SENSE = sensitivity encoding; SLF $=$ superior longitudinal fasciculus; $\mathrm{UNC}=$ uncinate fasciculus.
}

cal level and the course or modification of subcortical tracts. ${ }^{6,18,27,46-48}$ These techniques are particularly useful because they can help the surgeon in surgical planning and, when the data are loaded into the neuronavigational system ${ }^{45}$ and available intraoperatively, may provide intraoperative guidance as well. ${ }^{6,13,17,32}$ Specifically, DT imaging and FT are MR imaging techniques that allow the visualization of the amount and orientation of water diffusion along myelinated fibers and enable 3D reconstruction and visualization of white matter tracts. ${ }^{1,2,11,14,35,44,52,60}$ In addition, DT imaging-FT depicts the relationship of subcortical tracts with the tumor mass and the surrounding brain edema. ${ }^{12,20,34,50} \mathrm{~A}$ tract can be depicted as unchanged (when its course is not modified by the tumor or edema), dislocated, or infiltrated and/or interrupted (when the tract is in strict relationship with the mass or interrupted by it). ${ }^{25,57}$ These results are influenced by some technical factors,,$^{30,51}$ such as the fractional anisotropy threshold used for start and stop tracking, ${ }^{33,35,59}$ or by characteristics of the tumor, such as histology, edema, and location. $19,22,50,53,56$ 
We have previously described our experience with the use of DT imaging-FT during the surgical removal of gliomas located in eloquent areas, reporting the correlation between DT imaging-FT data and those obtained with DES applied at the subcortical level. ${ }^{5}$ In that preliminary experience, based on a limited group of patients with low- and high-grade gliomas, we showed that the location and course of the tract was dependent on tumor histological type and degree of infiltration; the tracts were usually at the tumor periphery in high-grade gliomas, or inside the tumor mass and highly infiltrated in low-grade gliomas. In addition, we also reported the percentage of correlation between some of the tracts, involved either in the motor or language function, as depicted by DT imaging-FT and DES findings. We concluded that the combination of DT imaging-FT and DES allowed accurate identification of eloquent fiber tracts and enhanced surgical performance and safety, maintaining a high rate of functional preservation. Furthermore, it decreased the duration of surgery, patient fatigue, and risk of intraoperative seizures. Since then, we have routinely used DT imaging-FT for reconstruction of subcortical tracts, involved either in the motor and language system, and correlate these data with those obtained intraoperatively by the aid of neurophysiology. This work describes such an experience, reporting the results in a larger group of patients, providing data pertaining to additional tracts involved in language, and discussing the advantage and pitfalls of our protocol.

For reasons of simplicity, we will focus on the correlation with neurophysiological data obtained with $60-\mathrm{Hz}$ stimulation, which is the most widely used in the clinical setting.

\section{Methods}

\section{Patient Population}

Between November 2005 and August 2009, 230 patients with gliomas in or within motor and/or language areas or pathways were admitted in our institutions. Age, sex, clinical history, and symptoms, as well as neurological status at admission, were registered. Preoperatively, all patients underwent a neuropsychological evaluation, baseline and volumetric MR imaging studies, fMR imaging, and DT imaging-FT. Surgery was performed in all patients with the aid of intraoperative cortical and subcortical mapping for motor and/or language functions. Volumetric scan analysis was used for establishing the exact tumor location and topography as well as the volume of the lesion. ${ }^{4}$

Tumor volume was calculated via a computerized system, using 3D axial FLAIR images (see below) for low-grade gliomas and contrast-enhanced T1-weighted MR images for high-grade gliomas. Histological type was classified according to the WHO brain tumor classification. Extent of resection was measured on postoperative volumetric FLAIR images (for low-grade gliomas) or post-contrast volumetric MR images (for high-grade gliomas) immediately after surgery or at 3 months and classified as previously reported. ${ }^{4,16,29}$
Surgical Procedures Including Cortical and Subcortical Mapping

Surgery was performed with the patient in a state of general (asleep) anesthesia when only the motor function was to be registered and monitored; alternatively, when language or visuospatial function also had to be monitored and tested, asleep-awake anesthesia was used. In these cases, a craniotomy exposing the cortex corresponding to the tumor area and a limited amount of the surrounding cortex was performed during the initial period of surgery, with the patient in a state of general anesthesia. Afterward, the dura was opened, the patient awakened, and cortical mapping performed. Multimodal electrophysiological monitoring was used throughout the entire duration of surgery. It included continuous EEG and ECoG recording along with a multichannel polygraphic recording of EMG responses (Comet system, Grass Technologies).

Electroencephalographic activity was recorded bilaterally by means of 4 subdermal needle electrodes, providing 4 bipolar leads. Electroencephalography was used to monitor brain activity when ECoG was not available, for example at the beginning and the end of surgery, and also to assess brain activity at sites distant from the operating field, such as in the contralateral hemisphere.

Electrocorticographic activity from a cortical region adjacent the area being stimulated was recorded by subdural strip electrodes (Cortical Strip Electrode, Integra LifeScience), in a monopolar array, referred to a midfrontal electrode. Cerebral activity was recorded with a bandpass of $1.6-320 \mathrm{~Hz}$, and displayed with a sensitivity of $50-100 \mu \mathrm{m} / \mathrm{cm}$ for EEG and $200-400 \mu \mathrm{m} / \mathrm{cm}$ for ECoG. Continuous electrocorticographic recording (Comet system, Grass Technologies) was used during the entire duration of the procedure to monitor the brain basal electrical activity, to define the working current (as that immediately below that which induced an afterdischarge), to monitor for the occurrence of afterdischarges, to monitor and adjust the level of anesthesia, or to detect seizures during resection.

For continuous monitoring of motor function, MEP recording was also performed. The train-of-5 technique, being introduced for surgery in anesthetized patients, has been described as sensitive to detect imminent lesions of the motor cortex and the pyramidal pathways. ${ }^{9}$ A strip electrode containing 4-8 electrodes was placed over the precentral gyrus. In awake patients a single stimulus or a double pulse stimulus (individual pulse width $0.3-0.5$ msec, anodal constant current stimulation; interstimulus interval $4 \mathrm{msec}$, stimulation close to motor threshold) was usually delivered. The muscle MEPs were recorded with either needle or-more convenient in awake patientssurface EMG electrodes. The MEP recording was usually alternated with direct cortical and subcortical motor mapping.

Cortical and subcortical mapping was performed with the use of an Orisis stimulator (Inomed), delivering biphasic square wave pulses, each phase lasting $1 \mathrm{msec}$, at $60 \mathrm{~Hz}$ in trains lasting 1 second for cortical mapping and 1-2 seconds for subcortical mapping. Subcortical mapping was alternated with resection. Subcortical mapping 
was performed by using the same current threshold applied for cortical mapping. Surgery was performed with the aid of a neuronavigation system (BrainLAB).

Motor mapping was performed with the aid of a continuous multichannel EMG recording, which was used throughout the entire procedure. Several separate muscles belonging to agonist or antagonist groups were monitored on either the contralateral or the ipsilateral side. Motor responses were collected via pairs of subdermal hooked needle electrodes inserted into contralateral muscles from face to foot. Each pair of electrodes recorded activity from 2 different muscles in the same body segment (for example, a flexor and an extensor muscle in the forearm) in order to sample as many muscles as possible. On average, 16 channels were used for each procedure. Most often, the muscles used included muscles from the face (upper and lower face), neck, arm, forearm, hand, upper leg, and lower leg. A computerized video image system was continuously coupled with the EMG recordings (Comet system, Grass Technologies) to monitor and record the motor activity. Motor activity was also evaluated clinically. In addition, in cases involving patients with a lesion in the dominant hemisphere, language mapping was performed. In such cases, patients underwent extensive preoperative language testing composed of a battery of tests aimed at evaluation of oral language production and comprehension together with repetition. ${ }^{4}$

Language dominance was evaluated by means of the Edinburgh Inventory Questionnaire and fMR imaging. The following tasks were performed: spontaneous speech; oral controlled association by phonemic cue; famous face naming; object picture naming; action picture naming; word comprehension; sentence comprehension; and transcoding tasks. In addition the token test, the digit span test, and counting were performed. Ideomotor apraxia and face apraxia were also assessed. The majority of the tests we used have been standardized on the normal population. Language was also evaluated during the entire duration of the surgical procedure by testing spontaneous speech.

\section{Neuroradiological Evaluation}

Magnetic resonance imaging was performed preoperatively on a Philips Intera 3.0-T system with a maximum field gradient strength of $80 \mathrm{mT} / \mathrm{m}$. Patients underwent standard MR imaging evaluation for morphological characterization of the lesions: conventional imaging consisted of an axial turbo spin echo T2-weighted sequence (TR $3800 \mathrm{msec}$, TE $85 \mathrm{msec}$, FOV $230 \mathrm{~mm}$, 22 slices, section thickness $5 \mathrm{~mm}$ with a 1-mm gap, matrix $512 \times 512$, SENSE reduction factor 1.5), a 3D axial FLAIR sequence (TR 10,000 msec, TE $110 \mathrm{msec}$, FOV $230 \mathrm{~mm}, 120$ slices, section thickness $1.5 \mathrm{~mm}$ with no gap, matrix $224 \times 256$, SENSE reduction factor 2), and a postcontrast inversion recovery T1-weighted sequence (TR $2000 \mathrm{msec}$, TE 10 msec, FOV $230 \mathrm{~mm}$, 22 slices, section thickness $5 \mathrm{~mm}$ with a 1 -mm gap, matrix $400 \times 512$, SENSE reduction factor 1.5).

\section{Diffusion Tensor Imaging Data Acquisition and FT Processing}

Diffusion tensor imaging data were obtained at $3 \mathrm{~T}^{21}$, ${ }^{37,41}$ using a single-shot echo planar imaging sequence (TR $8986 \mathrm{msec}$, TE $80 \mathrm{msec}$ ) with parallel imaging (SENSE reduction factor 2.5) ${ }^{23}$ Diffusion gradients were applied along 32 axes, using $b$ values of 0 and $1000 \mathrm{~mm}^{2} / \mathrm{second}$. An FOV of $240 \times 240 \mathrm{~mm}$ and a data matrix of $96 \times 96$ were used, resulting in isotropic voxel dimensions of 2.5 $\times 2.5 \times 2.5 \mathrm{~mm}$. The data were interpolated in plane to a matrix of $256 \times 256$ leading to a voxel size of $0.94 \times 0.94$ $\times 2.5 \mathrm{~mm}$. Fifty-six slices were obtained, with a thickness of $2.5 \mathrm{~mm}$ and no gap. The sequence was repeated twice and data were averaged offline to increase the signal-tonoise ratio; thus, total time for DT MR imaging was 10 minutes and 46 seconds. Three-dimensional fast field echo T1-weighted sequences (TR $8 \mathrm{msec}$, TE $4 \mathrm{msec}$, image resolution equal to DT imaging) were obtained for anatomical guidance.

Diffusion tensor imaging data sets were realigned offline on a PC workstation using the AIR (Automatic Image Registration) software to correct for artifacts due to rigid body movement during scan acquisition..$^{58}$

Deterministic tractography was performed in all patients with the aid of DTI Studio v2.4.01 software $(\mathrm{H}$. Jiang and S. Mori, Department of Radiology, Johns Hopkins University), obtaining main eigenvector and fractional anisotropy maps. ${ }^{43}$ Subcortical connections were reconstructed using the "fiber assignment by continuous tracking" (FACT) method..$^{33,36,59}$ A fractional anisotropy threshold of 0.1 and a turning angle $>55^{\circ}$ were used as criteria to start and stop tracking. Seeding ROIs for tractography were defined around areas of white matter that represent the brain regions that all the fibers of each tract must pass through to reach their cortical or subcortical end points. The ROIs were chosen, on the basis of previous anatomical knowledge, in sections perpendicular to the main course of the tracts. ${ }^{54}$

To reconstruct the CST, an ROI was placed on an axial section at the level of the subcortical white matter of the precentral gyri. For the IFO, ILF, and UNC, an ROI was placed on a coronal section at the level of the anterior part of the external capsule at the junction of the frontal and temporal lobes, where the 2 tracts run in contiguity.

To reconstruct the SLF, a first ROI was placed on a coronal section at the level of a high-anisotropy region lateral to the central part of the lateral ventricle; a second ROI was placed in a peritrigonal site at the level of the descending branch of the fascicle.

For all the tracts reconstructed, contaminating fibers were removed.

Volumetric precontrast T1-weighted or FLAIR images were coregistered to the mean of all diffusion weighted images using SPM2 software to obtain the superimposition of the white matter tracts on T1-weighted anatomical images. This allowed us to compare the trajectories of the tracts in the involved hemisphere with those of the contralateral (unaffected) hemisphere, and to evaluate the anatomical relationship between the tract and the tumor mass as well as the effect exerted by the tumor on the tract of interest. The DT imaging-FT data were saved in 
a compatible format (DICOM) to be transferred to the neuronavigational system using MEDx Software (Medical Numerics, Inc.).

\section{Correlation Between DT Imaging-FT Data and Perioperative Subcortical Motor and Language Findings}

As previously reported, the correlation between DT imaging-FT data and subcortical sites identified during perioperative DES was assessed at the time of surgery and postoperatively. At the time of surgery, the location of each subcortical site was recorded during the various phases of resection by the use of neuronavigation system into which the DT imaging-FT data fused with preoperative MR images were loaded. The distance between the point of response to DES and the tract border was measured on axial navigation images, and relationship between the two was graded as correspondent (at the tract border or within the tract), close (within $1 \mathrm{~cm}$ ), or distant $(>1 \mathrm{~cm})$. In addition, each subcortical site identified during subcortical mapping was marked with a sterile numbered tag and a digital picture of the surgical cavity was taken at the end of the resection. On the immediate postoperative MR images we evaluated the anatomical location of the subcortical pathways (the periphery of the surgical cavity, where the resection was stopped according to the functional responses elicited by intraoperative stimulation). ${ }^{16}$ This was accomplished by transferring immediate postoperative MR images obtained in each patient to a BrainLAB workstation and fusing them with preoperative MR images with which DT imaging-FT results for each fascicle had been merged. The existence of a correspondence between the location of a subcortical site identified by DES and that of the tract in DT imaging-FT images was considered only when the distance between the location of the subcortical site evoked by DES and identified intraoperatively by the use of neuronavigation system was graded as correspondent to the tract in axial neuronavigation images. In all the other cases, the correspondence was considered null.

\section{Statistical Analysis}

Data were analyzed by the use of Prism 4 for Macintosh (GraphPad Software, Inc).

Differences in the duration of resection expressed as means and SDs were evaluated with the Student t-test. Differences in percentages of patients with clinical and electrical evidence of seizure activity and differences in patient fatigue were studied with the Fisher exact test. Sensitivity and specificity of DT imaging-FT for the identification of subcortical tracts were calculated as previously reported. ${ }^{5}$

\section{Results and Discussion}

\section{Patient Population}

Two hundred and thirty patients were included. Of these, 176 had low-grade gliomas (oligodendrogliomas and astrocytomas) and 54 had high-grade gliomas (glioblastomas or anaplastic astrocytomas and oligodendrogliomas). As for location, 71 tumors were in the precen- tral lobe, 71 were rolandic, 18 parietal, 46 temporal, and 24 within the insula. In all patients we were able to reconstruct subcortical tracts. Depending on the location of the tumor and language dominance, various language tracts were reconstructed.

Results will be presented hereafter according to tumor histological type and motor and language tracts.

\section{Tumor Histological Type}

The analysis of the course of the tracts according to tumor histological type confirmed the results obtained in our previous work based on a smaller group of patients. In patients with high-grade gliomas, most of the tracts were located at the tumor periphery and were depicted as dislocated or infiltrated. No tract was found inside a tumor mass. Subcortical mapping demonstrated functional subcortical areas in the same location, specifically at the tumor border. In addition, when the tract was found to be infiltrated and/or interrupted, no responses were evoked by DES in the same areas. In patients with low-grade gliomas, DT imaging-FT showed that the tracts were mostly infiltrated and interrupted or dislocated by the tumor mass. In addition, in many patients large portions of the tracts were shown to be inside the tumor mass, in the same places where DES identified motor or language responses. Furthermore, when tracts were found interrupted inside the tumor mass, in some cases DES documented the presence of functional areas in the same areas. Globally considered, these data indicate that DT imaging and FT reconstruction are particularly useful in low-grade glioma surgery, where most of the tracts are inside the tumor mass. ${ }^{5,9,24}$ This is the reason why we preferentially performed DT imaging-FT in patients in whom preoperative MR images suggested a low-grade glioma; it was used in only selected cases in which preoperative images indicated a high-grade glioma.

\section{Motor Tracts}

Per our routine protocol, the CST was reconstructed in all 230 patients and tested intraoperatively by DES due to its close relationship with the tumor in 180 cases. In selected patients, additional tracts running in the premotor areas were also reconstructed, mainly for research purposes; these findings are not included in this work. As an exception, the course of the motor fibers belonging to the premotor facial areas were also reconstructed. These findings are reported under Language Tract.

In all the cases of precentral tumors, the CST was depicted either as unchanged (66\% of cases) or as dislocated posteriorly (34\% of tumors, all large lesions) (Table 1). In both conditions, subcortical stimulation located the tract at the posterior border of the tumor mass. Motor responses appeared as focal (few muscles) when the tract was stimulated in close vicinity to the surface, whereas responses to deep stimulation involved multiple muscle groups. The cortical or near-cortical stimulation always induced evident movements, while subcortical stimulation frequently induced muscle activation that was only detected by magnified EMG in the early stages of resection, when approaching the subcortical tracts, and that 


\section{Combined DTI and DES in gliomas}
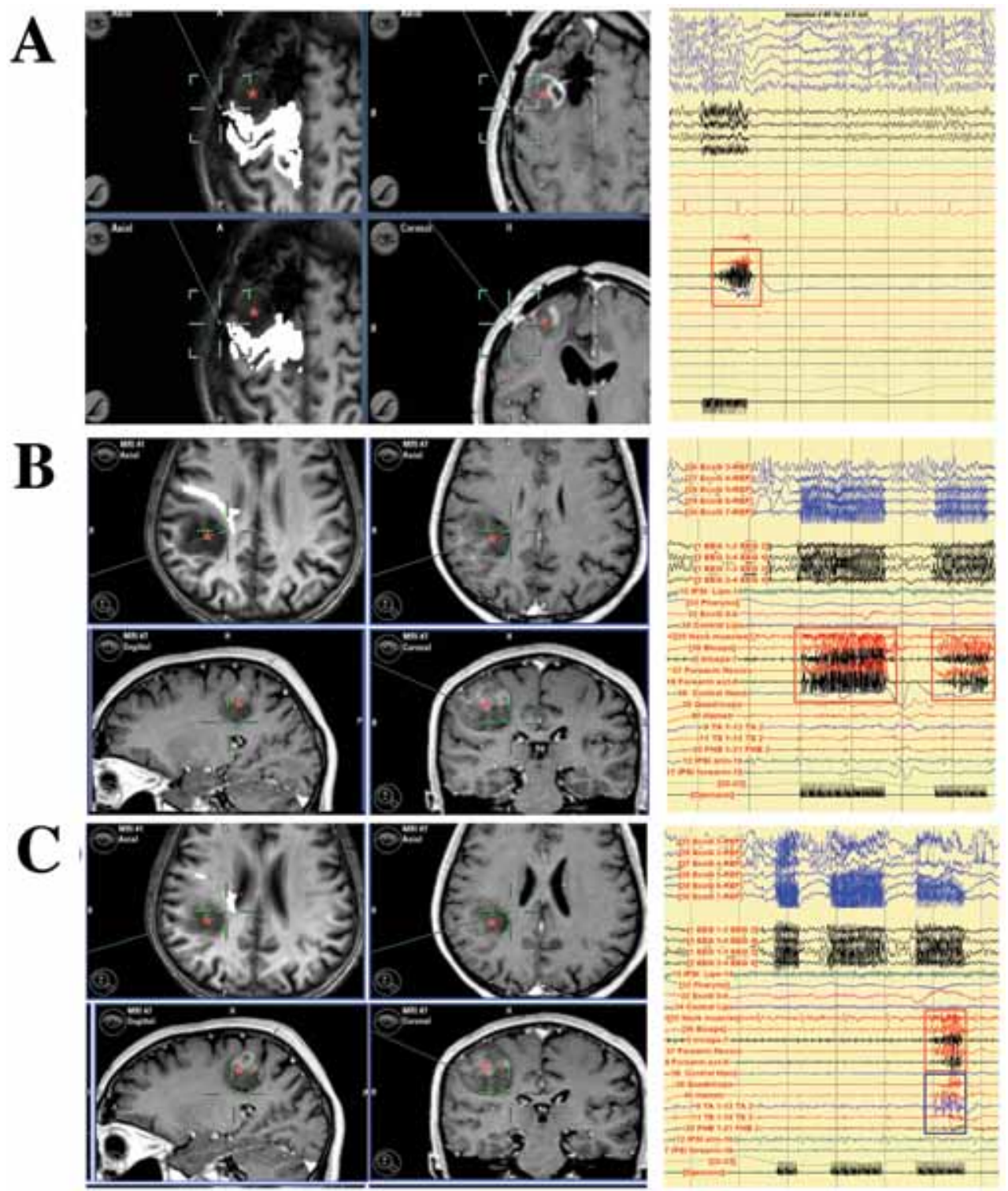

FIG. 1. Diffusion tensor imaging-FT and DES data for the CST obtained in patients with rolandic tumors. The left panels show intraoperative screen shots taken where DES located motor responses. The right panels show EMG responses evoked during subcortical stimulation. In cases of rolandic tumors (red asterisks), DT imaging reconstructed the CST (bright white tract) inside the tumor mass. Motor responses to DES appeared as focal (forearm muscles grouped in the red rectangle [A]) when the tract was stimulated in close vicinity to the surface and progressively affected multiple muscle groups with stimulation of the deeper portion of the tract (hand and arm [B]; arm and leg [C], grouped in the red and blue rectangles, respectively). For all shown cases, the stimulation point is indicated by the center of the green cursor.

became overt movements when the CST was reached. In all cases, subcortical DES located the CST where it was shown by DT imaging-FT, and therefore, the results were graded as correspondent.

In cases of rolandic tumors, DT imaging reconstructed the CST mainly inside the tumor mass (98\% of cases). In the majority of bulky tumors (92\%), the tract was displaced either anteriorly (22\%), or more frequently posteriorly (78\%), and highly infiltrated by the tumor mass (Fig. 1, Table 1). Less frequently, and in cases of highly infiltrating and diffuse low-grade gliomas, the tract was depicted inside the tumor mass and as highly infiltrated. In the first group of tumors, subcortical DES located the tract in the same position where it was depicted by DT imaging-FT (Fig. 1). Some discrepancies were observed in the superior portion of the tract, close to the cortical surface, where DT imaging-FT failed to reconstruct fibers and instead DES located motor responses. As we previously reported, even the placement of additional ROIs in those regions did not improve the fiber reconstruction. More problematic are the cases of highly diffuse low-grade gliomas, where DT imaging-FT usually reconstructed the tract as highly infiltrated and inside the tumor mass (Fig. 2). Particularly in those cases with a long history of seizures, and at the beginning of the resection, when $60-\mathrm{Hz}$ stimulation was applied over the regions of the tumor where DT 
TABLE 1: Corticospinal tract: preoperative DT imaging-FT findings, correspondence with intraoperative subcortical mapping, and postoperative deficits*

\begin{tabular}{|c|c|c|c|c|c|c|}
\hline \multirow{2}{*}{$\begin{array}{l}\text { Tumor } \\
\text { Location \& Preop } \\
\text { DTI-FT Findings } †\end{array}$} & \multirow{2}{*}{$\begin{array}{l}\text { No. of } \\
\text { Cases }\end{array}$} & \multirow{2}{*}{$\begin{array}{l}\text { No. of Cases w/ } \\
\text { Intraop Mapping } \ddagger\end{array}$} & \multicolumn{2}{|c|}{ Correspondence§ } & \multicolumn{2}{|c|}{ Deficitsף } \\
\hline & & & Yes & No & Early & Late \\
\hline \multicolumn{7}{|l|}{ rolandic (71 cases) } \\
\hline unchanged & 8 & 8 & 8 & 0 & 0 & 0 \\
\hline dislocated & 13 & 13 & 13 & 0 & 11 & 0 \\
\hline infiltrated & 50 & 50 & $50(12)^{\star *}$ & 0 & 50 & 0 \\
\hline \multicolumn{7}{|l|}{ precentral (71 cases) } \\
\hline unchanged & 47 & $24 \dagger \dagger$ & 24 & 0 & 0 & 0 \\
\hline dislocated & 24 & 24 & 24 & 0 & 23 & 0 \\
\hline infiltrated & 0 & - & - & - & - & - \\
\hline \multicolumn{7}{|l|}{ insular (24 cases) } \\
\hline unchanged & 14 & 0 & - & - & - & - \\
\hline dislocated & 10 & 10 & 10 & 0 & 10 & 0 \\
\hline infiltrated & 0 & - & - & - & - & - \\
\hline \multicolumn{7}{|l|}{ parietal (18 cases) } \\
\hline unchanged & 3 & 3 & 3 & 0 & 0 & 0 \\
\hline dislocated & 7 & 7 & 7 & 0 & 6 & 0 \\
\hline infiltrated & 8 & 8 & 8 & 0 & 8 & 0 \\
\hline \multicolumn{7}{|l|}{ temporal ( 46 cases) } \\
\hline unchanged & 28 & 0 & - & - & - & - \\
\hline dislocated & 18 & 10 & 10 & 0 & 1 & 0 \\
\hline infiltrated & 0 & - & - & - & - & - \\
\hline \multicolumn{7}{|c|}{$\begin{array}{l}\text { * Values represent numbers of cases. Abbreviation: }-=\text { not applicable. } \\
\dagger \text { The DT imaging-FT findings represent the modification of the tract in relationship to the tumor. } \\
\text { † The number of cases in which DT imaging-FT depicted the tract as adjacent to or inside the tumor mass and it was possible to } \\
\text { directly test the correspondence between DT imaging-FT and intraoperative subcortical mapping. } \\
\S \text { Correspondence between DT imaging and subcortical mapping with respect to the location of the tract. } \\
\text { † Early = } 3 \text { days after surgery; late = } 1 \text { month after surgery. } \\
\text { ** Includes } 12 \text { cases of highly diffuse low-grade gliomas in which the CST was depicted by DT imaging-FT as inside the tumor } \\
\text { mass and highly infiltrated and in which a good correspondence was reached by the combined use of monopolar and bipolar } \\
\text { stimulation. } \\
\dagger † \text { The number of precentral tumors in which the CST was depicted close to the posterior border of the tumor and therefore found } \\
\text { during surgery by DES. }\end{array}$} \\
\hline
\end{tabular}

imaging-FT depicted the location of the upper portion of the tract, it usually failed to locate overt motor responses. When the current intensity was progressively increased to induce responses, it usually resulted in seizures, without overt movements. In these cases, the electrical identification of the CST required the use of different modalities of stimulation, such as the monopolar stimulation, or alternatively, the initial resection could be performed under DT imaging-FT guidance. However, as in the previous cases, DT imaging-FT failed to show fibers close to the more lateral portion of the homunculus, probably due to the presence of crossing fibers that cannot be depicted by the simple tensor model here used for tractography, ${ }^{26,55}$ where DES (generally with monopolar stimulation) induced laryngeal or upper or lower face responses. When a portion of the tumor was removed, and the CST partially decompressed, the $60-\mathrm{Hz}$ stimulation again started to produce motor responses, usually in the same location where DT imaging-FT reconstructed the deeper portion of the CST.

In the cases of parietal tumors, DT imaging-FT usually located the CST at the anterior border of the tumor, and depicted either as unchanged when the tumor volume was small (16.7\% of cases), or dislocated anteriorly when the tumor was larger $(83.3 \%$, Table 1). Direct electrical stimulation located the tract in a similar position.

In cases of insular or temporal tumors, the CST was located at the medial and posterior border of the tumor, and depicted either as unchanged or dislocated (Table 1). The concordance was tested in a subgroup of patients in which the tract was depicted as close to the border of the tumor, where DES during surgery produced motor responses.

Considered together, these data indicate that there is usually a very high concordance between DT imagingFT data for CST and subcortical mapping, with the CST 


\section{Combined DTI and DES in gliomas}

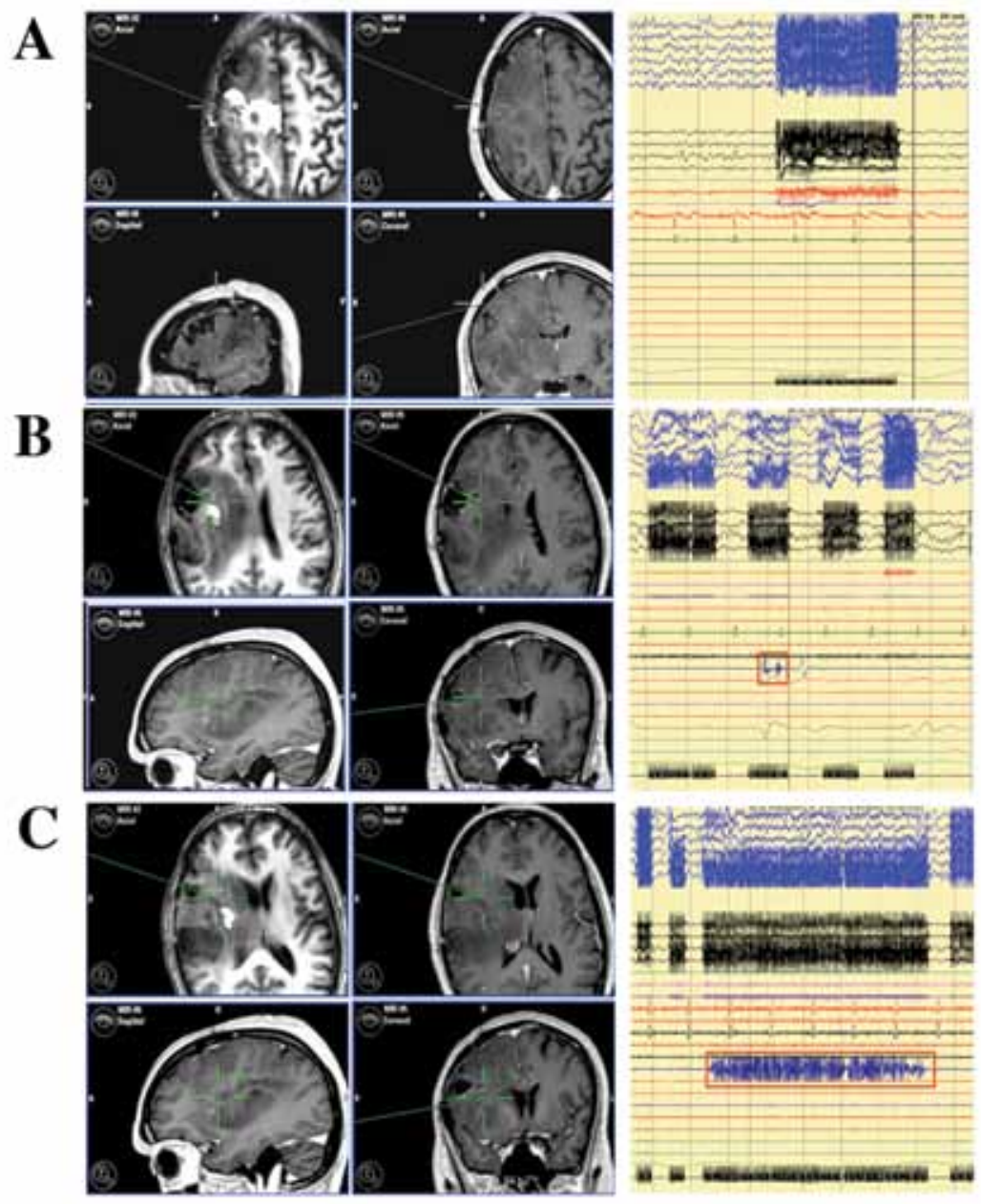

FIG. 2. Diffusion tensor imaging-FT and DES data for the CST in a case of diffuse low-grade glioma. The left panels show intraoperative screen shots taken where DES identified motor responses. The right panels show EMG responses evoked during subcortical stimulation. In this patient, DT imaging-FT reconstructed the tract as highly infiltrated and inside the tumor mass. In the upper portion of the tract, the $60-\mathrm{Hz}$ stimulation failed to induce overt motor responses, as shown in $\mathbf{A}$. When a portion of the tumor was removed and the CST partially decompressed, the $60-\mathrm{Hz}$ stimulation started again to produce motor responses with stimulation in the same location where DT imaging-FT reconstructed the deeper portion of the CST, as shown in the red rectangles in $\mathbf{B}$ and $\mathbf{C}$.

being located in the same position where it was depicted by DT imaging-FT. Some pitfalls may occur in a limited subgroup of highly diffuse low-grade gliomas located in the rolandic areas (12 cases in this study), in which DT imaging-FT may fail in reconstructing portions of CST, particularly in areas of extensive infiltration. In addition, in these particular cases, the electrical identification of the CST may be problematic and require the use of alternative stimulation modalities.

\section{Language Tracts}

We will initially present the results for the SLF and the IFO and then we will analyze the results for additional language tracts, such as UNC and ILF, separately.

The SLF and IFO were reconstructed in all patients with either temporal or frontal dominant tumors, given that these 2 tracts are essential for the preservation of language function.
The SLF is a large tract,$^{31}$ running from the parietal to the frontal lobe, mediating the phonemic component of language. It was depicted by DT imaging-FT as dislocated or infiltrated by the tumor, generally depending on histological type, size, and location of the tumor mass (Fig. 3, Table 2). The tract was usually unchanged or dislocated in patients with high-grade tumors, and infiltrated in those with low-grade gliomas. Large tumors dislocated or infiltrated the tract; smaller ones were more likely to leave the tract unchanged. When the SLF was found during surgery and stimulated with a $60-\mathrm{Hz}$ current, phonemic paraphasias were induced by stimulation in the same places where the tract was located by DT imaging-FT. As previously reported, the anatomical distribution of this tract is usually quite a bit larger than the functional distribution as identified by subcortical mapping. Therefore, a large part of the tract can be safely resected because it is not functional in terms of language. This is particularly true in patients with frontal and temporal tumors. In 

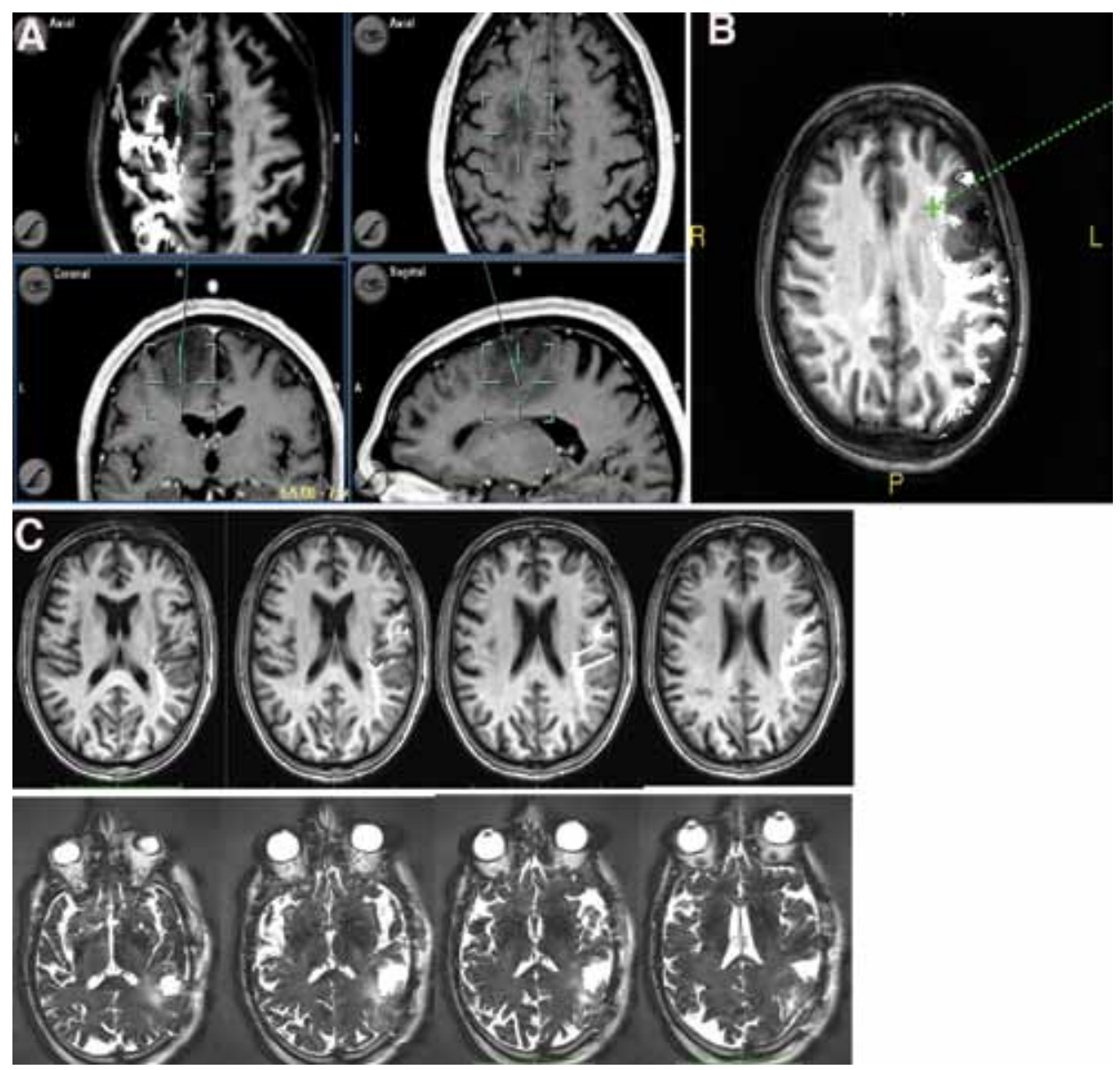

FIG. 3. Diffusion tensor imaging-FT reconstruction of the SLF. A: A case of a left frontal F1, F2 Grade Il oligodendroglioma. Diffusion tensor imaging-FT reconstructions of the SLF, highlighted as a bright white ROI, were fused with T1-weighted MR images. The tract constituted the lateral border of the tumor. Resection was performed with the aid of subcortical language mapping, which was continuously alternated with the tumor resection. Resection was stopped when language responses (phonemic paraphasias) were encountered. The intraoperative screen shots were taken where DES, performed in the site indicated by the center of the green cursors, identified such a response. B: A case of an F3 low-grade glioma. Diffusion tensor imaging-FT reconstruction of the SLF was fused with T1-weighted MR images. To visualize the portion of the tract located at the anterior and medial border of the tumor, an additional ROI was placed in the subcortical region of F3. Intraoperative DES induced phonemic paraphasias. The location of this site (green cross) was registered into the neuronavigation system at the time of surgery, as visualized by the intraoperative screen shot. C: A case of left temporal oligodendroglioma. Diffusion tensor imaging-FT reconstruction of the SLF was merged with T1-weighted MR images (upper panels). Postoperative T2-weighted MR images showed that the resection margins coincided with the location of the SLF (lower panels).

addition, in cases of low-grade gliomas involving the F3 gyrus, an additional ROI should be placed in the subcortical area of F3 to visualize the portion of the SLF tract that produces phonemic paraphasia in response to stimulation. This portion usually constituted the anterior and superior border of the tumor in our patients.

The IFO is a discrete tract, running from the occipital to the frontal lobe, and mediating the semantic component of language. The relationship between the tract and the tumor depends on the tumor's location, size, and histological type. The tract was depicted as unchanged or dislocated in cases of high-grade tumors, or as dislocated and/or infiltrated in cases of low-grade gliomas. Again, large tumors dislocated or infiltrated the tract; small tumors were more likely to leave the tract unchanged. It is to be stressed that the anatomical distribution of this tract is small and usually corresponds to the functional one de- picted by subcortical mapping (Fig. 4, Table 3). In fact, DES evoked no language disturbances in areas lacking fibers when the tract was depicted as interrupted inside the tumor mass. In only a few cases of frontal low-grade gliomas ( 9 of 76), where the tract was located inside the more anterior portion of the tumor mass and depicted as highly dislocated and infiltrated, a limited portion of the tract could be safely removed because it was not functional. In addition, some problems may occur for F3 low-grade gliomas in which DT imaging-FT may fail in reconstructing the more superior part of the tract at the inferior border of the tumor, when the tumor infiltration in this area is quite extensive.

These data indicate that the reconstruction of the IFO by DT imaging-FT is of particular use, because the results correlate closely with the results of functional mapping by DES. In contrast, the anatomical distribution of 


\section{Combined DTI and DES in gliomas}

TABLE 2: Superior longitudinal fasciculus in dominant-hemisphere tumors: preoperative DT imaging-FT findings, correspondence with intraoperative subcortical mapping, and postoperative deficits

\begin{tabular}{|c|c|c|c|c|c|c|}
\hline \multirow{2}{*}{$\begin{array}{l}\text { Tumor Location \& Preop } \\
\text { DTI-FT Findings }\end{array}$} & \multirow{2}{*}{$\begin{array}{l}\text { No. of } \\
\text { Cases }\end{array}$} & \multirow{2}{*}{$\begin{array}{c}\text { No. of Cases } \\
\text { w/ Intraop } \\
\text { Mapping }\end{array}$} & \multicolumn{2}{|c|}{ Correspondence } & \multicolumn{2}{|c|}{ Deficits } \\
\hline & & & Yes & No & Early & Late \\
\hline \multicolumn{7}{|l|}{ rolandic (30 cases) ${ }^{*}$} \\
\hline unchanged & 3 & 3 & 3 & 0 & 0 & 0 \\
\hline dislocated & 6 & 6 & 6 & 0 & 6 & 0 \\
\hline infiltrated & 21 & 21 & 21 & 0 & 21 & 0 \\
\hline \multicolumn{7}{|l|}{ precentral (40 cases) ${ }^{*}$} \\
\hline unchanged & 8 & 8 & 8 & 0 & 0 & 0 \\
\hline dislocated & 5 & 5 & 5 & 0 & 4 & 0 \\
\hline infiltrated & 27 & 27 & 27 & 0 & 26 & 0 \\
\hline \multicolumn{7}{|l|}{ insular (18 cases) ${ }^{*}$} \\
\hline unchanged & 1 & 0 & - & - & - & - \\
\hline dislocated & 6 & 6 & 6 & 0 & 6 & 0 \\
\hline infiltrated & 11 & 11 & 11 & 0 & 11 & 0 \\
\hline \multicolumn{7}{|l|}{ parietal (14 cases)* } \\
\hline unchanged & 0 & - & - & - & - & - \\
\hline dislocated & 4 & 4 & 4 & 0 & 2 & 0 \\
\hline infiltrated & 10 & 10 & 10 & 0 & 6 & 0 \\
\hline \multicolumn{7}{|l|}{ temporal (40 cases) ${ }^{*}$} \\
\hline unchanged & 0 & - & - & - & - & - \\
\hline dislocated & 18 & 18 & 18 & 0 & 14 & 0 \\
\hline infiltrated & 22 & 22 & 22 & 0 & 22 & 0 \\
\hline
\end{tabular}

* Number of patients with a left-hemisphere tumor in whom the tract was reconstructed.

SLF by DT imaging-FT is quite large, and particularly inside the tumor mass, a large part of the tract can be safely resected.

The UF was reconstructed in cases of insular tumors or frontal tumors, and in cases of temporal tumors. In insular tumors, the UF ran at the anterior border of the tumor mass and was depicted usually as dislocated and infiltrated in low-grade gliomas or anteriorly dislocated in high-grade tumors. When the UF was found during subcortical stimulation, phonemic paraphasia followed by speech arrest resulted from stimulation in the same areas where the tract was depicted by DT imaging-FT. In frontal tumors that extended posteriorly toward the anterior portion of the insula, the tract ran along the posterior inferior border, and it was dislocated posteriorly in $84 \%$ of cases. In cases of temporal tumors, the UNC was located in the medial anterior portion of the lobe, running rostrally. The identification of the tract both in DT imaging-FT maps and during surgery depended on tumor size. The tract could be easily identified as a separate fascicle in cases of small or medium-sized tumors, in which it usually runs anteriorly, medially, and inferiorly to the IFO. In cases of large tumors, DT imaging-FT showed that the tract was intermingled with the IFO, and during surgery it could not be identified, as it was "masked" by the IFO.

The ILF is a discrete fascicle running in the anterior and lateral portion of the temporal lobe, laterally and su- periorly to the IFO, close to the ventricle. The tract can be depicted as unchanged or dislocated or infiltrated depending on the size and histological type of the tumor. High-grade gliomas usually dislocated the tract, whereas low-grade gliomas infiltrated it. The ILF was identified as a separate fascicle in cases of small- or medium-sized tumors (Fig. 5). In large tumors, it was depicted as intermingled mainly along its posterior part with the IFO, from which it could not be functionally separated at the time of surgery. Functional results by others ${ }^{15,17,18}$ as well as by our group have shown that the tract can be safely resected during surgery, resulting in a transient speech disturbance that resolved in less than 2 weeks.

In cases of F3 or F2 frontal or insular tumors, we found it useful to reconstruct the course of the face premotor fibers. These are fibers running from the dorsal premotor cortex toward the Broca area, and their stimulation during surgery induced anarthria. As with the IFO and SLF, the maintenance of these fibers is essential for the preservation of language. Diffusion tensor imaging-FT depicted the course of these fibers at the posterior border of F3, F2, or insular tumors, where they were identified by DES (Fig. 6).

Clinical Significance of the Combined Use of DT Imaging-FT and Subcortical DES

Our experience with a large number of patients 


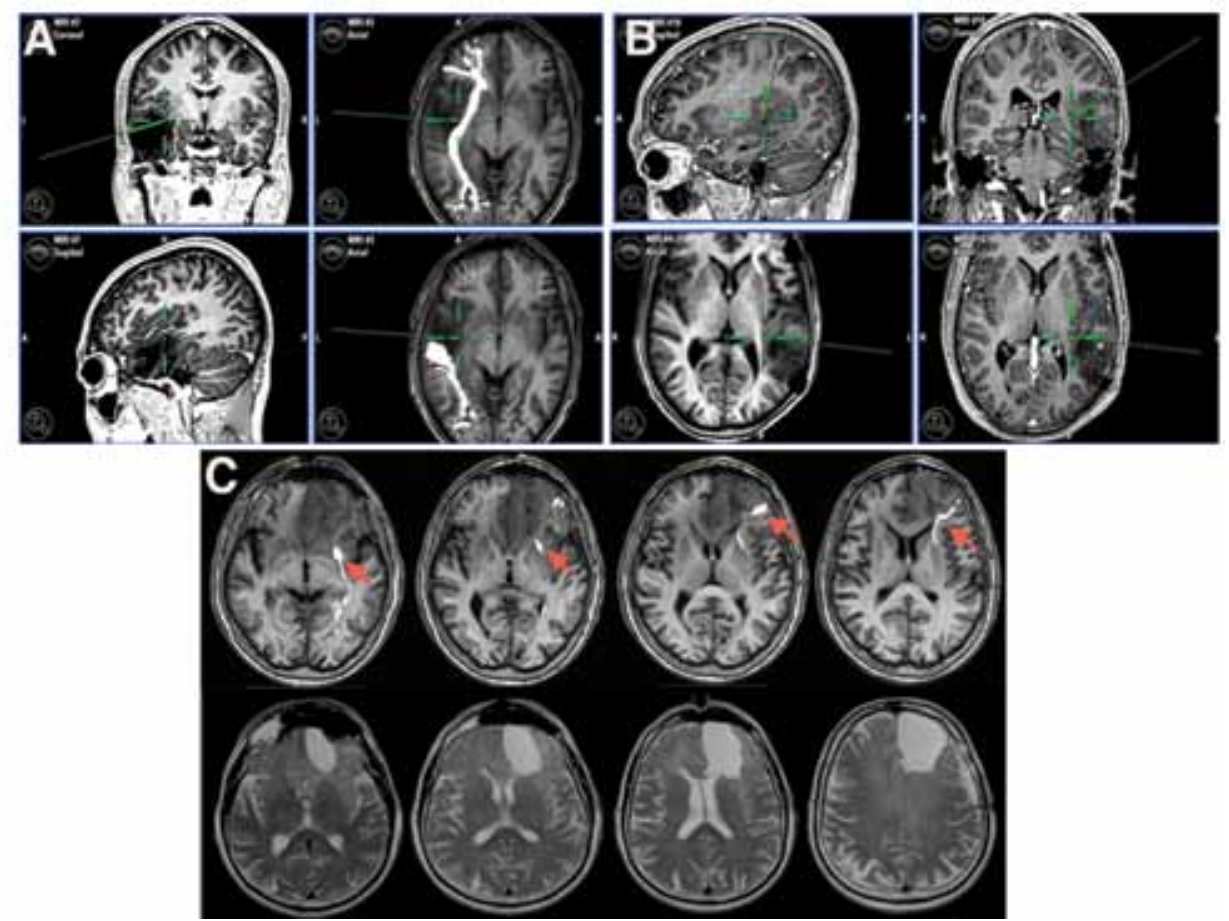

Fig. 4. Diffusion tensor imaging-FT reconstruction of the IFO. A and B: Cases of low-grade temporal oligodendrogliomas. Diffusion tensor imaging-FT reconstruction of the IFO was fused with postcontrast T1-weighted MR images and loaded into the neuronavigation system. The IFO, shown as a highlighted bright white ROI, was located at the superior medial border of the tumor, where DES evoked semantic paraphasias (DES sites were located at the center of the green cursors). C: A case of a left frontal oligodendroglioma. Diffusion tensor imaging-FT reconstruction of the IFO, which was merged with postcontrast T1-weighted MR images, showed that the tract was located at the posterior border of the tumor (upper panels, arrows). Postoperative T2-weighted MR images showed that the resection margins coincided posteriorly with the location of the IFO as shown by DT imaging-FT (lower panels).

showed that the combined use of DT imaging-FT and DES is a feasible approach that can be effectively and safely applied in routine clinical practice. Diffusion tensor imaging-FT, when used with a neuronavigation system, can help reduce the time required for surgery by helping the surgeon design the surgical approach as well as helping-at the time of the resection-to locate the tract where subcortical stimulation should be initiated. This may result in a reduction in the number of stimulations needed to safely identify a tract as well as a decrease in seizures and in patient fatigue. As already reported in limited cases, such as in diffuse low-grade gliomas, this combined approach may help at the beginning of the resection when the use of a $60-\mathrm{Hz}$ current may be problematic. In addition, in our experience it has also helped in a few cases in which surgery was performed under awake anesthesia, when at the end of a long procedure the patient's cooperation became suboptimal, rendering direct language guidance no longer useful. In these few cases, we were able to continue resection under the guidance of DT imaging-FT images until the position of subcortical tracts was encountered and to maintain the patients' functional integrity.

In our patient population, the combined use of DT imaging-FT and DES for identification of subcortical tracts during surgery was associated with immediate postoperative deficits in 72.2 and $84 \%$ of patients with lesions involving motor or language pathways, respec- tively, and these deficits lasted 1 week on average (Tables 1-3). Deficits were most likely to develop in those cases in which preoperative DT imaging showed the tracts as dislocated or infiltrated. At 1-month follow-up, 94\% of patients with a motor lesion had normal findings on motor function examination, and $96.8 \%$ of those with lesions involving speech areas or pathways had normal language function.

\section{Neuronavigation, Brain Shift, and Technical Performance} of Surgery

It is important to remember that DT imaging-FT provides anatomical information, whereas subcortical motor or language mapping provides functional information. This distinction affects the concordance between DT imaging-FT images and functional information obtained with subcortical mapping. This is of some importance for CST but is of particular relevance for language tracts, in which the anatomical distribution of the tract as depicted by DT imaging can be larger than the functional ones obtained with mapping. Therefore, portions of some tracts as depicted by DT imaging-FT can be removed because they are not involved in the function tested at that time.

As a whole, the data presented in the previous section indicate that the reconstruction by DT imaging-FT of specific tracts, such as the CST, IFO, SLF and face premotor fibers, and its availability at the time of surgery are of particular help, especially for resection of low-grade 


\section{Combined DTI and DES in gliomas}

TABLE 3: Inferior frontoccipital fasciculus in left-hemisphere tumors: preoperative DT imaging-FT findings, correspondence with intraoperative subcortical mapping, and postoperative deficits

\begin{tabular}{|c|c|c|c|c|c|c|}
\hline \multirow{2}{*}{$\begin{array}{c}\text { Tumor Location \& Preop } \\
\text { DTI-FT Findings }\end{array}$} & \multirow{2}{*}{$\begin{array}{l}\text { No. of } \\
\text { Cases }\end{array}$} & \multirow{2}{*}{$\begin{array}{c}\text { No. of Cases } \\
\text { w/ Intraop } \\
\text { Mapping }\end{array}$} & \multicolumn{2}{|c|}{ Correspondence } & \multicolumn{2}{|c|}{ Deficits } \\
\hline & & & Yes & No & Early & Late \\
\hline \multicolumn{7}{|l|}{ rolandic (40 cases) ${ }^{*}$} \\
\hline unchanged & 28 & 0 & - & - & - & - \\
\hline dislocated & 12 & 12 & 12 & 0 & 10 & 0 \\
\hline infiltrated & 0 & - & - & - & - & - \\
\hline \multicolumn{7}{|l|}{ precentral (40 cases) ${ }^{*}$} \\
\hline unchanged & 3 & 1 & 1 & 0 & 1 & 0 \\
\hline dislocated & 12 & 12 & 12 & 0 & 11 & 0 \\
\hline infiltrated & 25 & 25 & $25(9) \dagger$ & 0 & 25 & 0 \\
\hline \multicolumn{7}{|l|}{ insular (14 cases) } \\
\hline unchanged & 2 & 2 & 2 & 0 & 1 & 0 \\
\hline dislocated & 8 & 8 & 8 & 0 & 8 & 0 \\
\hline infiltrated & 4 & 4 & 4 & 0 & 4 & 0 \\
\hline \multicolumn{7}{|l|}{ parietal (12 cases) ${ }^{*}$} \\
\hline unchanged & 3 & 0 & - & - & - & - \\
\hline dislocated & 6 & 6 & 6 & 0 & 3 & 0 \\
\hline infiltrated & 3 & 3 & 3 & 0 & 3 & 0 \\
\hline \multicolumn{7}{|l|}{ temporal (40 cases) ${ }^{*}$} \\
\hline unchanged & 0 & - & - & - & - & - \\
\hline dislocated & 22 & 22 & 22 & 0 & 22 & 0 \\
\hline infiltrated & 18 & 18 & 18 & 0 & 18 & 0 \\
\hline
\end{tabular}

* Number of cases of left-hemisphere tumors in which the tract was reconstructed.

$\dagger$ Includes 9 cases of left precentral tumors (low-grade gliomas) in which it was possible to safely resect the anterior portion of a highly infiltrated IFO which was determined to be not functional by intraoperative subcortical mapping.

gliomas. Apart from the cases in which DT imaging-FT data can be obtained intraoperatively by the use of an intraoperative MR imaging system, ${ }^{38-40}$ in the majority of settings DT imaging-FT data are usually loaded into the neuronavigation system and fused with preoperative MR images. For a correct use of DT imaging-FT data in this setting, 2 points appear as critical: the transfer of the data to the neuronavigation system, and the use of technical adjustments during surgery to maintain global accuracy of the information. . $8,82,42^{2}$

With respect to the first point, in our center DT imaging-FT data are saved in a compatible format (DICOM) by using MEDx Software (Medical Numerics, Inc.); this allows the images to be transferred and loaded into the neuronavigation system. The BrainLAB neuronavigation system performed an automatic coregistration between DT imaging-FT datasets and the preoperative MR images acquired with skull fiducials by means of a voxelby-voxel intensity-matching nonlinear algorithm. As an estimate of the clinical navigation accuracy, the target registration error localizing a separate fiducial, which was not used for registration, was also determined; this target registration error was less than $2 \mathrm{~mm}$. Furthermore, repeated landmark checks were performed during surgery to ensure overall ongoing clinical navigation accuracy.

With respect to the second point, the maximal ac- curacy of the neuronavigation system should be maintained to reduce the problem of brain shift: 1) Repeated landmark checks should be performed during surgery to ensure overall ongoing clinical navigation accuracy. 2) Craniotomy size should be limited to the minimum necessary to expose the tumor area and a limited portion of the surrounding brain. 3) Resection should be performed in a manner that ensures maintenance of maximal accuracy of the information. In cases of frontal tumors located in the proximity of the CST, resection was started from the posterior border of the tumor, where the CST was located, and after its identification the tract was followed inside the tumor mass. Afterward the remaining anterior part of the tumor was removed. Similarly, in cases of parietal tumors, resection was started from the anterior border following the same principle. In any case, during resection of temporal or insular tumors, or at the end of the resection of large tumors, even when these suggestions were carefully followed, the amount of brain shift can become substantial, and the accuracy of the information provided by the neuronavigation system may be limited..$^{38-40}$

\section{Conclusions}

Globally considered, our data indicate the usefulness 

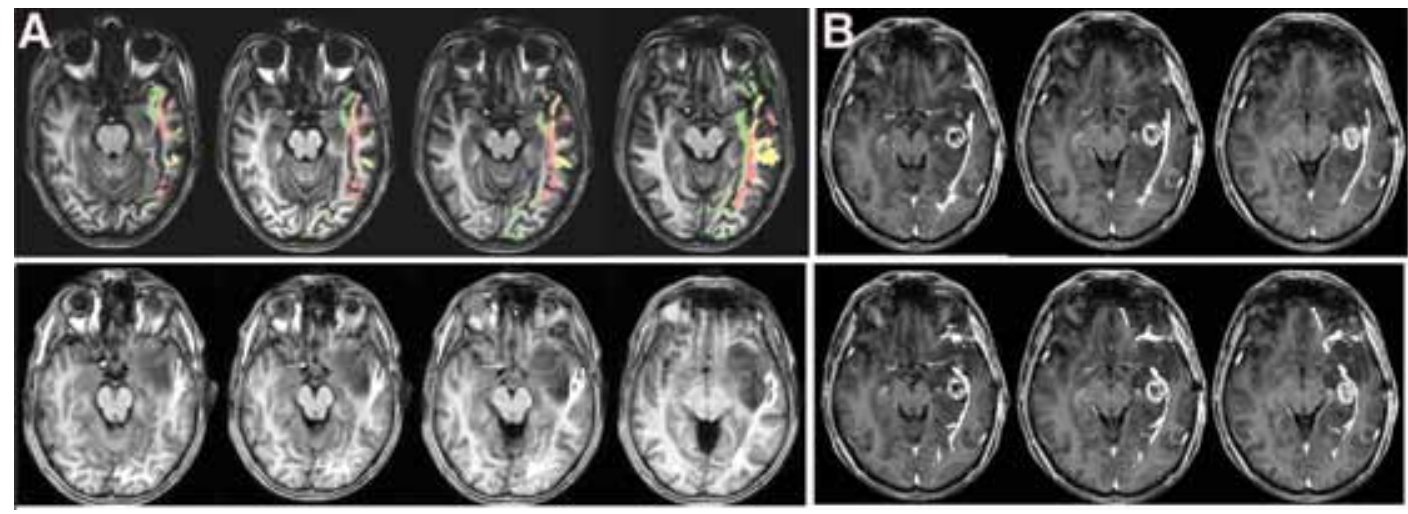

Fig. 5. Diffusion tensor imaging-FT reconstruction of the ILF. A: A case of a low-grade temporal oligodendroglioma. Diffusion tensor imaging-FT reconstructions of the IFO (green ROIs), ILF (red RO/s), and SLF (yellow ROls) were merged with T1-weighted MR images (results shown in upper panels). Images with only the DT imaging-FT reconstruction of the ILF (white $R O / s$ ) were fused with T1-weighted MR images (results show in lower panels). Both sets of images show that the tracts were inside the tumor mass and can be depicted as separate fascicles. B: A case of a large left temporal high-grade glioma. The DT imaging-FT reconstruction of the ILF is shown in the upper panels and that of the IFO in the lower panels. The ILF and IFO (white ROls, upper and lower panels, respectively) were fused with postcontrast T1-weighted MR images. In this case, the ILF was intermingled with the IFO, and they could not be functionally separated during surgery.

of the routine combined use of DT imaging-FT and subcortical mapping, particularly in patients with low-grade gliomas. These tumors display an infiltrative modality of growth, along short and long connecting fibers, and visualizing the trajectory of the tracts is important for planning and performing surgery. When used in combination with subcortical mapping, DT imaging-FT offers the opportunity to quickly find the fibers associated with motor or language functions during surgery. The clinical relevance of this combined approach comes from the
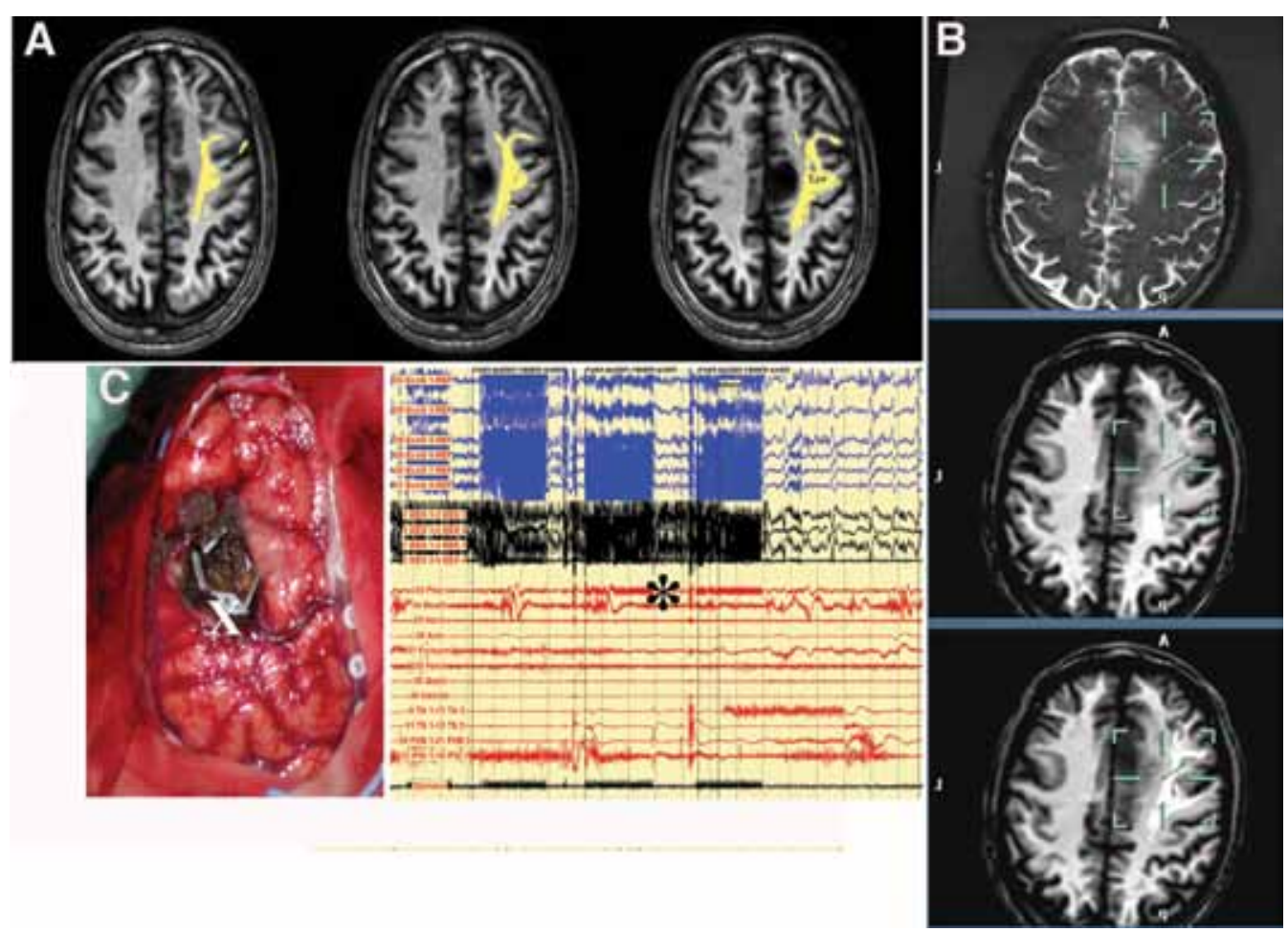

FIG. 6. Diffusion tensor imaging-FT reconstruction of the face premotor fibers. A: A case of a left-hemisphere oligodendroglioma in which DT imaging-FT reconstruction of the face premotor fibers (yellow RO/s) was merged with T1-weighted MR images. The fibers were located at the lateral border of the tumor. B: A similar case of a left-hemisphere oligodendroglioma, in which DES, performed at the center of the green cursors, located the fibers intraoperatively, in the same position as depicted by DT imaging-FT as white ROIs (lower panel). The middle panel shows the DT imaging-FT reconstruction of the CST. C: The left panel is an intraoperative picture of the surgical field. The white $X$ indicates the position of the fibers at the posterior border of the surgical cavity. The right panel reports the EMG findings. The black asterisk indicates the activation of the upper and lower lips, which resulted in speech arrest (anarthria). 
fact that it further enhances surgical safety, maintaining a high rate of functional preservation. Careful use requires the knowledge of its limitations, mainly the occurrence of brain shift.

\section{Disclosure}

This work was supported by grants from AIRC (Associazione Italiana Ricerca sul Cancro) and Fondazione Italo Monzino to Dr. Bello.

Author contributions to the study and manuscript preparation include the following. Conception and design: L Bello, A Falini. Acquisition of data: L Bello, A Castellano, E Fava, G Casaceli, M Riva, A Falini. Analysis and interpretation of data: L Bello, A Castellano, G Casaceli, M Riva, SM Gaini. Drafting the article: L Bello, E Fava. Critically revising the article: L Bello, A Castellano, G Casaceli, G Scotti, A Falini. Reviewed final version of the manuscript and approved it for submission: L Bello, G Scotti, A Falini.

\section{References}

1. Basser PJ, Pajevic S, Pierpaoli C, Duda J, Aldroubi A: In vivo fiber tractography using DT-MRI data. Magn Reson Med 44:625-632, 2000

2. Beaulieu C: The basis of anisotropic water diffusion in the nervous system - a technical review. NMR Biomed 15:435455, 2002

3. Bello L, Acerbi F, Giussani C, Baratta P, Taccone P, Songa $\mathrm{V}$, et al: Intraoperative language localization in multilingual patients with gliomas. Neurosurgery 59:115-125, 2006

4. Bello L, Gallucci M, Fava M, Carrabba G, Giussani C, Acerbi $F$, et al: Intraoperative subcortical language tract mapping guides surgical removal of gliomas involving speech areas. Neurosurgery 60:67-82, 2007

5. Bello L, Gambini A, Castellano A, Carrabba G, Acerbi F, Fava E, et al: Motor and language DTI Fiber Tracking combined with intraoperative subcortical mapping for surgical removal of gliomas. Neuroimage 39:369-382, 2008

6. Berman J: Diffusion MR tractography as a tool for surgical planning. Magn Reson Imaging Clin N Am 17:205-214, 2009

7. Berman JI, Berger MS, Chung SW, Nagarajan SS, Henry RG: Accuracy of diffusion tensor magnetic resonance imaging tractography assessed using intraoperative subcortical stimulation mapping and magnetic source imaging. J Neurosurg 107:488-494, 2007

8. Berman JI, Berger MS, Mukherjee P, Henry RG: Diffusiontensor imaging-guided tracking of fibers of the pyramidal tract combined with intraoperative cortical stimulation mapping in patients with gliomas. J Neurosurg 101:66-72, 2004

9. Bertani G, Fava E, Casaceli G, Carrabba G, Casarotti A, Papagno $\mathrm{C}$, et al: Intraoperative mapping and monitoring of brain functions for the resection of low-grade gliomas: technical considerations. Neurosurg Focus 27(4):E4, 2009

10. Black PM: Brain tumors. Part 1. N Engl J Med 324:14711476, 1991

11. Bürgel U, Amunts K, Hoemke L, Mohlberg H, Gilsbach JM, Zilles K: White matter fiber tracts of the human brain: threedimensional mapping at microscopic resolution, topography and intersubject variability. Neuroimage 29:1092-1105, 2006

12. Clark CA, Barrick TR, Murphy MM, Bell BA: White matter fiber tracking in patients with space-occupying lesions of the brain: a new technique for neurosurgical planning? Neuroimage 20:1601-1608, 2003

13. Coenen VA, Krings T, Mayfrank L, Polin RS, Reinges MH, Thron A, et al: Three-dimensional visualization of the pyramidal tract in a neuronavigation system during brain tumor surgery: first experiences and technical note. Neurosurgery 49:86-93, 2001

14. Conturo TE, Lori NF, Cull TS, Akbudak E, Snyder AZ, Shimony JS, et al: Tracking neuronal fiber pathways in the living human brain. Proc Natl Acad Sci U S A 96:10422-10427, 1999

15. Duffau H: The anatomo-functional connectivity of language revisited. New insights provided by electrostimulation and tractography. Neuropsychologia 46:927-934, 2008

16. Duffau H, Capelle L, Sichez N, Denvil D, Lopes M, Sichez JP, et al: Intraoperative mapping of the subcortical language pathways using direct stimulations. An anatomo-functional study. Brain 125:199-214, 2002

17. Duffau H, Thiebaut de Schotten M, Mandonnet E: White matter functional connectivity as an additional landmark for dominant temporal lobectomy. J Neurol Neurosurg Psychiatry 79:492-495, 2008

18. Ellmore TM, Beauchamp MS, O’Neill TJ, Dreyer S, Tandon $\mathrm{N}$ : Relationships between essential cortical language sites and subcortical pathways. J Neurosurg 111:755-766, 2009

19. Goebell E, Paustenbach S, Vaeterlein O, Ding XQ, Heese O, Fiehler J, et al: Low-grade and anaplastic gliomas: differences in architecture evaluated with diffusion-tensor MR imaging. Radiology 239:217-222, 2006

20. Hendler T, Pianka P, Sigal M, Kafri M, Ben-Bashat D, Constantini $\mathrm{S}$, et al: Delineating gray and white matter involvement in brain lesions: three-dimensional alignment of functional magnetic resonance and diffusion-tensor imaging. J Neurosurg 99:1018-1027, 2003

21. Hunsche S, Moseley ME, Stoeter P, Hedehus M: Diffusiontensor MR imaging at 1.5 and 3.0 T: initial observations. Radiology 221:550-556, 2001

22. Inoue T, Ogasawara K, Beppu T, Ogawa A, Kabasawa H: Diffusion tensor imaging for preoperative evaluation of tumor grade in gliomas. Clin Neurol Neurosurg 107:174-180, 2005

23. Jaermann T, Crelier G, Pruessmann KP, Golay X, Netsch T, van Muiswinkel AM, et al: SENSE-DTI at 3 T. Magn Reson Med 51:230-236, 2004

24. Jbabdi S, Mandonnet E, Duffau H, Capelle L, Swanson KR Pélégrini-Issac $\mathrm{M}$, et al: Simulation of anisotropic growth of low-grade gliomas using diffusion tensor imaging. Magn Reson Med 54:616-624, 2005

25. Jellison BJ, Field AS, Medow J, Lazar M, Salamat MS, Alexander AL: Diffusion tensor imaging of cerebral white matter: a pictorial review of physics, fiber tract anatomy, and tumor imaging patterns. AJNR Am J Neuroradiol 25:356-369, 2004

26. Jones DK: Determining and visualizing uncertainty in estimates of fiber orientation from diffusion tensor MRI. Magn Reson Med 49:7-12, 2003

27. Kamada K, Todo T, Masutani Y, Aoki S, Ino K, Morita A, et al: Visualization of the frontotemporal language fibers by tractography combined with functional magnetic resonance imaging and magnetoencephalography. J Neurosurg 106: 90-98, 2007

28. Kamada K, Todo T, Masutani Y, Aoki S, Ino K, Takano T, et al: Combined use of tractography-integrated functional neuronavigation and direct fiber stimulation. J Neurosurg 102:664-672, 2005

29. Keles GE, Lundin DA, Lamborn KR, Chang EF, Ojemann G, Berger MS: Intraoperative subcortical stimulation mapping for hemispherical perirolandic gliomas located within or adjacent to the descending motor pathways: evaluation of morbidity and assessment of functional outcome in 294 patients. J Neurosurg 100:369-375, 2004

30. Kinoshita M, Yamada K, Hashimoto N, Kato A, Izumoto S, Baba T, et al: Fiber-tracking does not accurately estimate size of fiber bundle in pathological condition: initial neurosurgical 
experience using neuronavigation and subcortical white matter stimulation. Neuroimage 25:424-429, 2005

31. Makris N, Kennedy DN, McInerney S, Sorensen AG, Wang R, Caviness VS Jr, et al: Segmentation of subcomponents within the superior longitudinal fascicle in humans: a quantitative, in vivo, DT-MRI study. Cereb Cortex 15:854-869, 2005

32. Mikuni N, Okada T, Enatsu R, Miki Y, Hanakawa T, Urayama $\mathrm{S}$, et al: Clinical impact of integrated functional neuronavigation and subcortical electrical stimulation to preserve motor function during resection of brain tumors. J Neurosurg 106:593-598, 2007

33. Mori S, Crain BJ, Chacko VP, van Zijl PC: Three-dimensional tracking of axonal projections in the brain by magnetic resonance imaging. Ann Neurol 45:265-269, 1999

34. Mori S, Frederiksen K, van Zijl PC, Stieltjes B, Kraut MA, Solaiyappan M, et al: Brain white matter anatomy of tumor patients evaluated with diffusion tensor imaging. Ann Neurol 51:377-380, 2002

35. Mori S, Kaufmann WE, Davatzikos C, Stieltjes B, Amodei L, Fredericksen $\mathrm{K}$, et al: Imaging cortical association tracts in the human brain using diffusion-tensor-based axonal tracking. Magn Reson Med 47:215-223, 2002

36. Mori S, van Zijl PC: Fiber tracking: principles and strategiesa technical review. NMR Biomed 15:468-480, 2002

37. Nagae-Poetscher LM, Jiang H, Wakana S, Golay X, van Zijl PC, Mori S: High-resolution diffusion tensor imaging of the brain stem at 3 T. AJNR Am J Neuroradiol 25:1325-1330, 2004

38. Nimsky C, Ganslandt O, Fahlbusch R: Implementation of fiber tract navigation. Neurosurgery 58:ONS292-ONS304, 2006

39. Nimsky C, Ganslandt O, Hastreiter P, Wang R, Benner T, Sorensen AG, et al: Intraoperative diffusion-tensor MR imaging: shifting of white matter tracts during neurosurgical procedures-initial experience. Radiology 234:218-225, 2005

40. Nimsky C, Ganslandt O, Hastreiter P, Wang R, Benner T, Sorensen $\mathrm{AG}$, et al: Preoperative and intraoperative diffusion tensor imaging-based fiber tracking in glioma surgery. Neurosurgery 56:130-138, 2005

41. Okada T, Miki Y, Fushimi Y, Hanakawa T, Kanagaki M, Yamamoto A, et al: Diffusion-tensor fiber tractography: intraindividual comparison of 3.0-T and 1.5-T MR imaging. Radiology 238:668-678, 2006

42. Okada T, Mikuni N, Miki Y, Kikuta K, Urayama S, Hanakawa $\mathrm{T}$, et al: Corticospinal tract localization: integration of diffusion-tensor tractography at 3-T MR imaging with intraoperative white matter stimulation mapping-preliminary results. Radiology 240:849-857, 2006

43. Pajevic S, Pierpaoli C: Color schemes to represent the orientation of anisotropic tissues from diffusion tensor data: application to white matter fiber tract mapping in the human brain. Magn Reson Med 42:526-540, 1999

44. Pierpaoli C, Jezzard P, Basser PJ, Barnett A, Di Chiro G: Diffusion tensor MR imaging of the human brain. Radiology 201:637-648, 1996

45. Roux FE, Ibarrola D, Tremoulet M, Lazorthes Y, Henry P, Sol JC, et al: Methodological and technical issues for integrating functional magnetic resonance imaging data in a neuronavigational system. Neurosurgery 49:1145-1157, 2001

46. Schiffbauer H, Berger MS, Ferrari P, Freudenstein D, Rowley HA, Roberts TP: Preoperative magnetic source imaging for brain tumor surgery: a quantitative comparison with intraoperative sensory and motor mapping. J Neurosurg 97:13331342,2002
47. Schiffbauer H, Ferrari P, Rowley HA, Berger MS, Roberts TP: Functional activity within brain tumors: a magnetic source imaging study. Neurosurgery 49:1313-1321, 2001

48. Schonberg T, Pianka P, Hendler T, Pasternak O, Assaf Y: Characterization of displaced white matter by brain tumors using combined DTI and fMRI. Neuroimage 30:1100-1111, 2006

49. Skirboll SS, Ojemann GA, Berger MS, Lettich E, Winn HR: Functional cortex and subcortical white matter located within gliomas. Neurosurgery 38:678-685, 1996

50. Stadlbauer A, Ganslandt O, Buslei R, Hammen T, Gruber S, Moser E, et al: Gliomas: histopathologic evaluation of changes in directionality and magnitude of water diffusion at diffusion-tensor MR imaging. Radiology 240:803-810, 2006

51. Stadlbauer A, Nimsky C, Buslei R, Salomonowitz E, Hammen $\mathrm{T}$, Buchfelder M, et al: Diffusion tensor imaging and optimized fiber tracking in glioma patients: histopathologic evaluation of tumor-invaded white matter structures. Neuroimage 34:949-956, 2007

52. Stieltjes B, Kaufmann WE, van Zijl PC, Fredericksen K, Pearlson GD, Solaiyappan M, et al: Diffusion tensor imaging and axonal tracking in the human brainstem. Neuroimage 14:723-735, 2001

53. Tropine A, Vucurevic G, Delani P, Boor S, Hopf N, Bohl J, et al: Contribution of diffusion tensor imaging to delineation of gliomas and glioblastomas. J Magn Reson Imaging 20:905912,2004

54. Wakana S, Jiang H, Nagae-Poetscher LM, van Zijl PC, Mori S: Fiber tract-based atlas of human white matter anatomy. Radiology 230:77-87, 2004

55. Wiegell MR, Larsson HB, Wedeen VJ: Fiber crossing in human brain depicted with diffusion tensor MR imaging. Radiology 217:897-903, 2000

56. Wieshmann UC, Clark CA, Symms MR, Franconi F, Barker GJ, Shorvon SD: Reduced anisotropy of water diffusion in structural cerebral abnormalities demonstrated with diffusion tensor imaging. Magn Reson Imaging 17:1269-1274, 1999

57. Witwer BP, Moftakhar R, Hasan KM, Deshmukh P, Haughton V, Field A, et al: Diffusion-tensor imaging of white matter tracts in patients with cerebral neoplasm. J Neurosurg 97:568-575, 2002

58. Woods RP, Grafton ST, Holmes CJ, Cherry SR, Mazziotta JC: Automated image registration: I. General methods and intrasubject, intramodality validation. J Comput Assist Tomogr 22:139-152, 1998

59. Xue R, van Zijl PC, Crain BJ, Solaiyappan M, Mori S: In vivo three-dimensional reconstruction of rat brain axonal projections by diffusion tensor imaging. Magn Reson Med 42:1123-1127, 1999

60. Yamada K, Kizu O, Mori S, Ito H, Nakamura H, Yuen S, et al: Brain fiber tracking with clinically feasible diffusion-tensor MR imaging: initial experience. Radiology 227:295-301, 2003

Manuscript submitted October 15, 2009.

Accepted December 7, 2009.

Address correspondence to: Lorenzo Bello, M.D., Neurochirurgia, Dipartimento di Scienze Neurologiche, Università degli Studi di Milano, Fondazione Ospedale Maggiore Policlinico, Mangiagalli e Regina Elena, Via Francesco Sforza 35, 20122, Milano, Italy. email: lorenzo.bello@unimi.it. 